\title{
Risk of overtreatment with current peak flow criteria in self-management plans
}

\author{
W.R. Douma*, H.A.M. Kerstjens*, J.M. Rooyackers', G.H. Koëter*, \\ D.S. Postma*, Dutch CNSLD Study Group
}

Risk of overtreatment with current peak flow criteria in self-management plans. W.R. Douma, H.A.M. Kerstjens, J.M. Rooyackers, G.H. Koëter, D.S. Postma, Dutch CNSLD Study Group. @ERS Journals Ltd 1998.

ABSTRACT: Home peak expiratory flow (PEF) measurements have become the cornerstone of asthma self-management plans. However, the cut-off values for changing treatment have not been formally tested. This study focusses on the possible overtreatment brought about by the different cut-off values and denominators currently employed.

Data from 133 clinically stable asthmatic patients from a $2.5 \mathrm{yr}$ follow-up study were analysed.

The results showed that strict adherence to current criteria would lead to severe overtreatment, with up to $30 \%$ of clinically stable patients crossing into the lowest (red) zone at least once a year when personal best is the denominator and when it has not been limited to a defined time of day or to defined prior bronchodilator use. As expected, the passage of clinically stable patients into the lower zones became less frequent when cut-off values were sharpened and when time- and treatment-specific PEFs were used as the denominators.

Strict adherence to commonly used peak expiratory flow cut-off values would lead to considerable overtreatment. In order to avoid overtreatment, the morning peak expiratory flow before any (bronchodilator) treatment should be related to the personal best peak expiratory flow measured under the same conditions. The choice of the right cut-off value will also depend on studies being performed to test the amount of undertreatment with a given value.

Eur Respir J 1998; 12: 848-852.

In the last two decades there has been an increasing interest in self-management plans for patients with asthma [1-12]. Self-management plans are helpful to patients in understanding the chronic and variable nature of asthma and to monitor their disease. These plans enable patients to identify the earliest signs of deterioration and to act quickly to regain control [3]. Many plans have been developed, but there is no uniformity in criteria on when to consult a doctor or to change medication. Changes in management are usually based on patient-initiated contact, standardized symtom scores and/or peak expiratory flow (PEF) measurements. The latter is the only objective parameter in this respect and is used as a key parameter in most self-management plans. Usually, PEF values have been divided into three zones, each zone requiring a specific therapeutic approach. Communally used PEF zones are: 1) $>80 \%$, 2) between 60 and $80 \%$, and 3) $<60 \%$, measured on 1 day and expressed as a percentage of the personal best PEF or the predicted PEF value. The two lowest zones require a change in management. Cut-off values of 70 and $50 \%$ have also been employed. The clinical effectiveness of this division into zones depends largely on the choice of the PEF cut-off values, since these values determine both undertreatment and overtreatment. Therefore, it is important to develop acceptable PEF criteria in order to act accurately at the right time and to refrain from action
*Dept of Pulmonology, University Hospital Groningen, The Netherlands. +Dept of Pulmonology, Dekkerswald University of Nijmegen, The Netherlands

Correspondence: D.S. Postma

University Hospital Groningen

Dept of Pulmonology

Hanzeplein 1

9713 GZ Groningen

The Netherlands

Fax: 503619320

Keywords: Asthma peak expiratory flow self-management plans

Received: July 101997

Accepted after revision March 161998 when the disease is stable. Up to now, the cut-off values have been chosen rather arbitrarily and have not been formally studied in longitudinal follow-up studies in patients with asthma.

In this study the impact of advised PEF cut-off values on asthma treatment was evaluated in clinically stable patients with airways hyperresponsiveness and reversible airways obstruction. The study focused specifically on the prevalence of overtreatment in relation to the cut-off values in PEF for decisions on change in management. Furthermore, an evaluation was made of the change in prevalence of overtreatment when the lowest zone had to be scored on 2 successive days instead of 1 day. Finally, the study investigated whether effects on overtreatment changed when using different criteria for the personal best value, or measuring on specific times of the day, and when measuring PEF before or after bronchodilators.

\section{Patients and methods}

For this study data were used from a randomized, double-blind, multicentre clinical study in asthma and chronic obstructive pulmonary disease (COPD), from which the asthmatic subgroup was taken. The main goal of this study was to compare the effect of three treatment regimes 
( $\beta$-agonists plus either placebo, anticholinergic agents or corticosteroids, all by inhalation) on the long-term course and outcome of the disease [13]. The study included 274 patients 18-60 yrs old, with moderate to severe airways obstruction and hyperresponsiveness, at six university pulmonary outpatient clinics. The patients were selected if their baseline forced expiratory volume in one second (FEV 1 ) was $>1.2 \mathrm{~L}$, and between 4.5 and 1.64 sD below the predicted value, or if the ratio of their FEV1 to the inspiratory vital capacity was $>1.64$ SD below the predicted level; the provocative concentration of inhaled histamine causing a $20 \%$ fall in FEV1 (PC20) needed to be $<8 \mathrm{mg} \cdot \mathrm{mL}^{-1}$. Patients were excluded if they had a history of occupational asthma or other serious diseases (e.g. tubercu-losis, myocardial infarction, or malignancies). They were not allowed to use oral corticosteroids, $\beta$-blockers, nitrates, anticoagulants or antibiotics on a maintenance base. The study protocol was approved by the Medical Ethics Committees of all participating centres. All patients gave written informed consent.

For the analyses presented here the patients with asthma were selected, i.e. with hyperresponsiveness and reversible airways obstruction (bronchodilator response Š9\% pred) [14]. In total, 165 participants fulfilled these criteria. Former analysis had shown no difference in lung function, hyperresponsiveness, symptoms and PEF at baseline or at follow-up between the groups using only bronchodilators (terbutaline $500 \mu \mathrm{g}$ q.i.d. in combination with placebo or in combination with ipratropium bromide $40 \mu \mathrm{g}$ q.i.d.). Therefore, these two treatment arms were grouped in the analyses, leaving a group with inhaled corticosteroids (terbutaline $500 \mu \mathrm{g}$ q.i.d. in combination with beclomethasone $200 \mu \mathrm{g}$ q.i.d.) and a group with bronchodilators only.

\section{Peak expiratory flow measurements}

Patients were followed up for 2.5 yrs and were asked to record PEF values at home for 14 consecutive days before each three-monthly visit. For optimal use of the miniWright peak flow meter (Clement Clarke International, London, UK), all patients received a standardized instruction at the outpatient clinic and techniques were thoroughly checked at each subsequent visit. To enhance further the PEF card completion, patients were reminded by telephone or letter to fill in the cards, several weeks prior to each visit. PEF measurements were performed three times daily: in the morning upon rising, both before (PEFm-pre) and 10 min after study medication (PEFm-post) and in the afternoon before the evening meal $(\mathrm{PEFa})$. The highest value of three measurements at each time was recorded. Patients were asked to mark the values if bronchodilator therapy was used $<4 \mathrm{~h}$ before the measurement and these values were excluded from the analyses. In this report, PEF data were used only when measured in a clinically stable period. Therefore, PEF data were excluded when measured within a period of 2 weeks before starting a course of prednisolone and 5 weeks afterwards. A course of prednisolone was given in case of exacerbation, which was defined by increased complaints of cough, wheezing, dyspnoea and/or the need for rescue medication (salbutamol $400 \mu \mathrm{g}$ when needed) in excess of four additional rotacaps a day. The decision to start prednisolone was not based on PEF values.
PEF cards were only accepted when at least 9 of the 14 days were filled in. The analyses were limited to those patients with acceptable PEF diary cards at visit 1 (after 3 months of treatment) and with one or more acceptable diary cards from visit 2 (6 months of treatment). Data from 32 asthmatics could not be used in the analyses because of this criterion. These asthmatics were comparable to those included in the analyses with respect to age, sex, smoking habits, FEV1\% pred, bronchodilator response and $\mathrm{PC} 20$.

\section{Peak expiratory flow criteria}

PEF values were categorized using a three-coloured zonal system, analogous to a traffic light [3]. The analyses focused on the yellow and red zone, because these zones require a change in management as proposed by the guidelines for asthma management. PEF values were expressed both as a percentage of the predicted value and as a percentage of the personal best value $(\mathrm{PB})$. The $\mathrm{PB}$ was taken from the 14 days prior to visit 1,3 months after starting the study medication and, thus, when the corticosteroid-induced improvement in airway calibre had taken place [13]. Different criteria for the PB value were tested: $\mathrm{PB}$ values irrespective of time of day or prior use of bronchodilators $(\mathrm{PB} \Sigma)$ and time-specific personal best values (PBTS) taking into account the time of day and prior use of medication (PB of the morning PEF before the use of bronchodilators ( $\mathrm{PB}$ m-pre), $\mathrm{PB}$ morning postbronchodilator (PBm-post), and $\mathrm{PB}$ afternoon $(\mathrm{PB})$ ). The following cutoff values were analysed: the yellow zone between 60 and $80 \%$, and between 50 and $70 \%$ of both personal best (\% PB) and predicted PEF (\% PEFpred) and the red zone $<60 \%$ or $50 \%$ of PB or PEFpred, measured on 1 day or on 2 successive days.

\section{Bronchodilator response}

FEV1 was performed with calibrated water-sealed spirometers according to standardized guidelines [15]. At least three reproducible values (i.e. with $<5 \%$ difference among the recordings) were obtained, the highest value being used in the analyses. At baseline, the bronchodilator response was performed by measuring FEV 1 before and $20 \mathrm{~min}$ after $1,000 \mu \mathrm{g}$ terbutaline from a metered-dose inhaler administered through a $750 \mathrm{~mL}$ spacer device (Nebuhaler; Astra Pharmaceuticals, Rijswijk, the Netherlands). The bronchodilator response was expressed as a percentage of predicted FEV1 [14].

\section{Histamine challenge test}

Histamine challenge tests were performed with a $2 \mathrm{~min}$ tidal breathing method $[16,17]$. Results were expressed as PC20. For analysis, a value of 0.015 was assigned to each patient who responded to saline or to the lowest concentration of histamine $\left(0.03 \mathrm{mg} \cdot \mathrm{mL}^{-1}\right)$. During the follow-up, a value of 64 was assigned when FEV1 did not decrease by $20 \%$ from baseline after the highest concentration of histamine $\left(32 \mathrm{mg} \cdot \mathrm{mL}^{-1}\right)$.

\section{Allergy}

Skin-test reactivity to 12 common aeroallergens was measured intradermally. A positive skin test was defined as a mean wheal size $>0.7$ times the histamine wheal size [18]. 


\section{Data analysis}

Calculations with $\mathrm{PC} 20$ were performed using the base2 logarithm ( $\left.\log _{2} \mathrm{PC} 20\right)$, as this reflects doubling doses and normalized distributions. Skew of the distributions was assessed with Kolmogorov-Smirnov tests in order to decide whether to apply parametric (Student's t-test) or nonparametric techniques (Mann-Whitney U-test). Chisquared tests were used for dichotomous variables.

\section{Results}

Table 1 shows the baseline characteristics of both groups. In the corticosteroid group there were 43 participants, with mean (SD) age of 38 (12) yrs, 58\% of whom were male. In the bronchodilator group there were 90 participants, with a mean age of 38 (13) yrs, $60 \%$ of whom were male. Although PEF values tended to be larger at baseline in the corticosteroid group, there were no statistically significantly differences between the two groups of patients. After 3 months of therapy, PEF variation was smaller and $\mathrm{PB}$ values were higher in the corticosteroid group than in the bronchodilator group $(\mathrm{p}<0.05)$.

Figures 1 and 2 show the percentages of patients in both groups who had a PEF value in the yellow zone or red zone for 1 or more days per year with the cut-off values for PEF and different ways of expressing PEF. It should be emphasized that data were used only when patients were clinically stable (see Methods). When using the morn-ing PEF before bronchodilator use (m-pre) expressed as a percentage of $\mathrm{PB} \Sigma, 70 \%$ of the clinically stable asthmatics in the corticosteroid group (fig. 1) experienced at least 1 day per year in the yellow zone and 30\% 1 day in the red zone.

Table 1. - Baseline characteristics

\begin{tabular}{|c|c|c|c|c|}
\hline \multirow[b]{2}{*}{ Patients $n$} & \multicolumn{2}{|c|}{$\begin{array}{c}\text { Inhaled } \\
\text { corticosteroids }\end{array}$} & \multicolumn{2}{|c|}{$\begin{array}{c}\text { Bronchodilators } \\
\text { only }\end{array}$} \\
\hline & 43 & & 90 & \\
\hline Male \% & 58 & & 60 & \\
\hline Age yrs & 38 & (11.9) & 38 & (12.7) \\
\hline Current smokers $\%$ & 33 & & 26 & \\
\hline Allergic \% & 77 & & 79 & \\
\hline FEV $1 \%$ pred & 65.7 & (14.1) & 60.7 & $(15.6)$ \\
\hline$\Delta \mathrm{FEV}_{1} \%$ pred & 17.2 & (6.1) & 17.9 & $(6.7)$ \\
\hline $\log _{2} \mathrm{PC} 20$ histamine $\mathrm{mg} \cdot \mathrm{mL}^{-1}$ & -2.12 & $(1.82)$ & -2.45 & $(2.12)$ \\
\hline Geometric mean & 0.23 & & 0.18 & \\
\hline \multicolumn{5}{|l|}{ At baseline } \\
\hline Mean PEFm-pre L.s-1 & 399 & (134) & 364 & (94) \\
\hline Mean PEFm-post $\mathrm{L} \cdot \mathrm{s}^{-1}$ & 480 & (135) & 438 & (99) \\
\hline Mean PEFa L $\cdot \mathrm{s}^{-1}$ & 459 & (132) & 419 & (97) \\
\hline Mean PEF variation ${ }^{\dagger} \%$ & 17.9 & $(10.8)$ & 18.0 & (9.6) \\
\hline \multicolumn{5}{|l|}{ After 3 months } \\
\hline $\mathrm{PBm}$-pre $\mathrm{L} \cdot \mathrm{s}^{-1}$ & 493 & $(133) *$ & 411 & $(101)$ \\
\hline $\mathrm{PBm}$-post $\mathrm{L} \cdot \mathrm{s}^{-1}$ & 529 & $(118)^{*}$ & $=469$ & (93) \\
\hline $\mathrm{PBa} L \cdot \mathrm{s}^{-1}$ & 531 & $(116)^{*}$ & $=472$ & (99) \\
\hline $\mathrm{PB} \Sigma \mathrm{L} \cdot \mathrm{S}^{-1}$ & 539 & $(115)^{*}$ & $=486$ & (96) \\
\hline PEFpred L.S $\mathrm{s}^{-1}$ & 507 & & 509 & \\
\hline Mean PEF variation $\%$ & 11.6 & $(8.7)^{*}$ & 19.3 & $(9.8)$ \\
\hline
\end{tabular}

Values are shown as mean (SD). FEV1: forced expiratory volume in one second; $\mathrm{PC} 20$ : provocative concentration causing a $20 \%$ fall in FEV1; PEF: peak expiratory flow; : absolute difference in the morning and evening PEF/mean; m-pre: morning prebronchodilator; m-post: morning postbronchodilator; a: afternoon; PB: personal best; $\mathrm{PB} \Sigma$ : overall personal best irrespective of time of day and prior use of bronchodilators; $\triangle \mathrm{FEV} 1$ : change in FEV 1 after $1,000 \mu \mathrm{g}$ terbutaline. *: $\mathrm{p}<0.05$ by Student's t-test.

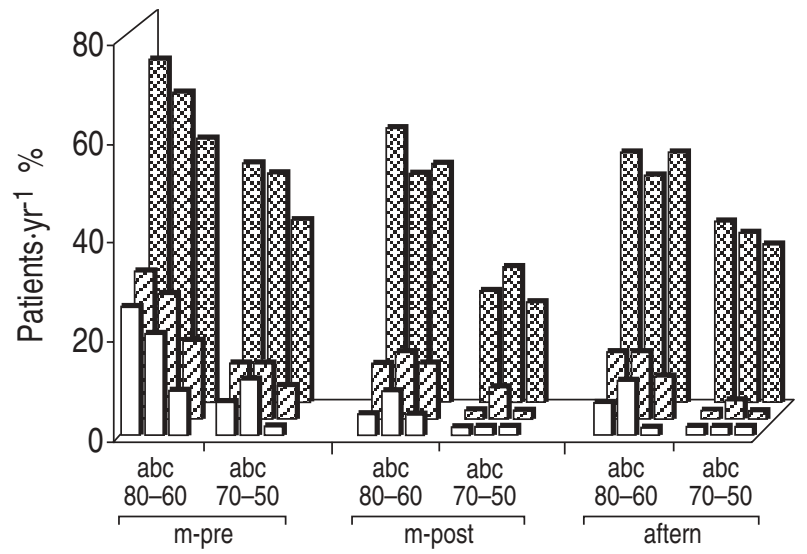

Fig. 1. - Percentage of clinically stable asthmatics in the inhaled corticosteroid group with peak expiratory flow (PEF) values in the yellow or red zone for $\breve{S} 1$ days $\cdot \mathrm{yr}^{-1}$. Different cut-off values are displayed. $\square: 2$

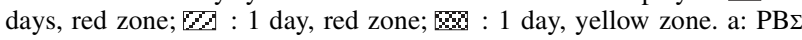
(overall personal best PEF); b: predicted PEF; c: PBTs (time-specific personal best PEF, accounting for time of day and prior use of bronchodilators). m-pre: morning PEF prebronchodilator; m-post: morning PEF postbronchodilator; aftern: afternoon PEF.

When the same cut-off value had to be exceeded on 2 successive days, $26 \%$ of all patients experienced at least one period per year in the red zone. In the group with bronchodilators only (fig. 2), these values were 99, 62 and $47 \%$ respectively.

As expected, when the cut-off values were sharpened from 80 to $70 \%$ and from 60 to $50 \%$ for the yellow and red zone, respectively, the percentages of the clinically stable patients with 1 or more days in the yellow and red zone were considerably lower. The percentages for the group with inhaled corticosteroids were $49 \%$ in the yellow zone, $12 \%$ in the red zone (measured on 1 day) and $7 \%$ (measured on 2 consecutive days), when PEF was expressed as a percentage of $\mathrm{PB} \Sigma$. In the group with bronchodilators these values were 83,36 , and $20 \%$, respectively.

The percentages of individuals in the yellow and red zone were highest when analysing the morning PEF prebronchodilator instead of the morning PEF postbronchodilator or the afternoons PEF. This was true for both

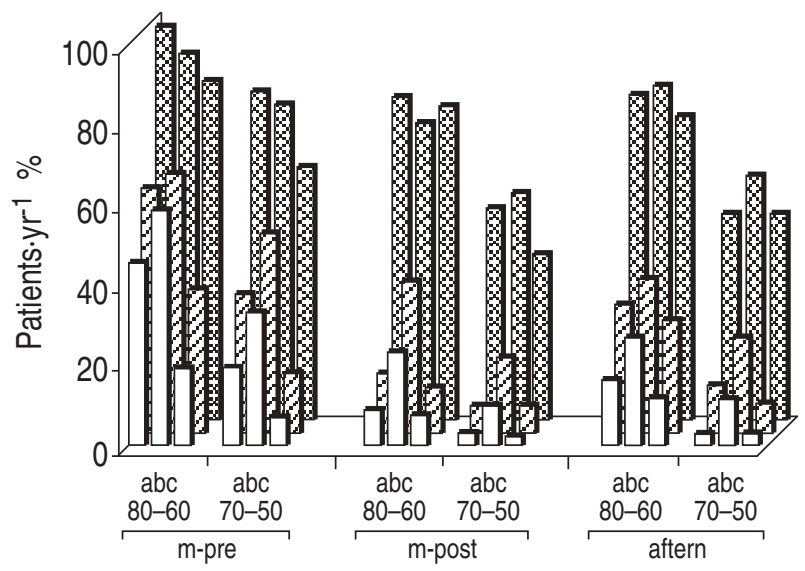

Fig. 2. - Percentage of clinically stable asthmatics in the bronchodilator group with peak expiratory flow (PEF) values in the yellow or red zone for $>1$ days $\cdot \mathrm{yr}^{-1}$. Different cut-off values are displayed. $\square: 2$ days, red zone; $Q: 1$ day, red zone; $\$: 1$ day, yellow zone. a: PB $\Sigma$ (overall personal best PEF); b: predicted PEF; c: PBTs (time-specific personal best PEF, accounting for time of day and prior use of bronchodilators). m-pre: morning PEF prebronchodilator; m-post: morning PEF postbronchodilator; aftern: afternoon PEF. 
treatment groups and irrespective of the expressions of the cut-off values. The percentages of patients in the yellow or red zone depend largely on the expressions of the cut-off values, and especially so for the morning PEF values before medication. Expressing PEF values as a percentage of PBTS, i.e. taking into account the time of the day and prior use of bronchodilators, resulted in the lowest percentages. Using $80 \%$ and $60 \%$ ( 1 day and 2 days) as cut-off values for the morning PEF prebronchodilator, $54 \%$ of the clinically stable patients were in the yellow zone, $16 \%$ in the red zone for 1 day and $9 \%$ in the red zone for 2 days in the group with inhaled corticosteroids, and 37, 7 and $2 \%$, respectively, using 70 and $50 \%$ ( 1 day and 2 days) as cutoff values.

For the group with bronchodilators only, using cut-off values 80 and $60 \%$, these percentages were 86,37 and $20 \%$, respectively, and 64,16 and $8 \%$ respectively when using 70 and $50 \%$ as cut-off values.

\section{Discussion}

For this study, a three-coloured zone system was used, analogous to a traffic light, as recommended in current guidelines for asthma management. The zones were separated by means of different cut-off values in PEF, as used in the various guidelines and publications [12]. Patients with a PEF value in the green zone do not need to change their medication. Patients with PEF values in the yellow zone usually have to start inhaled corticosteroids or increase the dose of inhaled corticostroids and bronchodilators. Finally, when a PEF value crosses the red zone border patients have to contact a doctor and/or use an oral corticosteroid course, depending on the guidelines to which they adhere.

The data show that strict adherence to PEF criteria in self-management plans would lead to considerable overtreatment. Overtreatment is believed to be the case since the present analyses were restricted to patients in clinically stable periods, i.e. PEF measurements were not made within a period of 2 weeks before starting a course of prednisolone and 5 weeks afterwards. The use of morning PEF before medication to assess whether an individual was in the yellow or red zone gave the highest level of overtreatment when these values were related to the overall personal best value, i.e. irrespective of the time of day and prior use of bronchodilators. Expressing the morning PEF before medication as a percentage of the time-specific $\mathrm{PB}$, i.e. taking into account both the time of day and prior use of medication, led to a substantial decrease in overtreatment. As expected, sharpening the cut-off criteria by reducing the border for the yellow zone from 80 to $70 \%$ and for the red zone from 60 to $50 \%$ also reduced the prevalence of overtreatment. Generally, overtreatment was slightly higher when using a cut-off value of $60 \%$ measured at 2 days than one of $<50 \%$ for one day. Finally, the use of cut-off values of 2 days $<50 \%$ of $\mathrm{PB} \Sigma$ (personal best value irrespective of time of day or prior use of bronchodilators), PEFpred or PBTS (time-specific personal best value taking into account both the time of day and prior use of medication) resulted in the lowest prevalence of overtreatment.

Cut-off values in self-management plans have been chosen rather arbitrarily and yet the effectiveness of the self-management plans depends to an important degree on the choice of these cut-off values. Since the cut-off values change the treatment plan for an individual patient, they determine both undertreatment and overtreatment. However, there are no firm data for the choice of these cut-off values and there has been no investigation into how the PEF should be expressed, i.e. as a percentage of predicted or as a percentage of the PB value, and how PB PEF should be calculated. PEF values are generally expressed as a percentage of their overall $\mathrm{PB}$ value (irrespective of both the time of day and prior use of bronchodilators) or as a percentage of the predicted value [19]. The advantage of the predicted value is that this value is fixed, irrespective of the time of day, use of medication or state of disease. There are, however, at least two disadvantages: asthmatics with more severe disease will continue to increase their medication, if 70 or $80 \%$ predicted would be unachievable, and predicted values of PEF are much less accurate than, for instance, predicted FEV1.

The concept of using the PB value is, in the authors opinion, more realistic. Usually, the PB has been defined as the individual's highest value, irrespective of both the time of day and prior use of bronchodilators. These more or less undefined or variable conditions under which PB values are established could lead to more frequent passing into the yellow and red zones. For instance, in asthmatic patients, PEF values have a diurnal variation, with the lowest values in the morning and the highest values in the afternoon. As a consequence, PEF measured in the morning will more often be in the yellow zone or red zone than PEF in the afternoon, when referred to the same personal best $\mathrm{PB} \Sigma$ or PEFpred at both times. PEF assessed before inhaling a bronchodilator will also more often be in the yellow or red zone than PEF measured after bronchodilator intake. This study has confirmed these theoretical assertions, in that PB values in the afternoon were higher than those measured in the morning (prebronchodilator). Relating PEF, measured at different times of the day, with the $\mathrm{PB} \Sigma$ gave the highest rate of overtreatment when using the morning PEF without prior use of medication. A general lowering of cut-off values would certainly temper overtreatment, but is a rather crude solution and could lead to a dangerous undertreatment. A better solution may be to relate measured peak flows to personal best values measured at the same time of the day. Furthermore, peak flows assessed before inhaling a bronchodilator should be related to personal best values that were also assessed before a bronchodilator. Treatment of patients with asthma consists of bronchodilators on demand or, in moderate to severe asthmatics, inhaled corticosteroids with bronchodilators on demand. From this, it can be stated that the PEF in the morning before medication would be the most useful parameter in self-management plans, because this value is least influenced by variable prior use of bronchodilators.

The data show that further reduction in overtreatment can be obtained by lowering the cut-off values from 80 $60 \%$ to $70-50 \%$ of PBTS, as well as by extension of the number of days to crossing the red zone from 1 to 2 successive days. Generally, overtreatment was more pronounced in patients using bronchodilators only than in patients with bronchodilators in combination with inhaled corticosteroids. Although clinically stable, the group with bronchodilators only were not optimally treated. According to current guidelines on asthma treatment, these patients should be treated with inhaled corticosteroids. Therefore, the term overtreatment should preferably be restricted to patients receiving inhaled corticosteroids. Patients in this 
study had a mean $\mathrm{PC}_{2} 0$ of $0.20 \mathrm{mg} \cdot \mathrm{mL}^{-1}$ and a mean FEV1 of $62 \%$ pred and therefore had mild to moderately severe asthma. Whether the results also apply to more severe patients remained to be determined. The data are in accordance with those of GiBSon et al. [20], who demonstrated in a small group of asthmatics that current PEF criteria have a high failure rate.

A critical issue is how to define clinically stable, and any chosen definition will influence the percentage overtreatment found in a study. There are no accepted or even formulated criteria of clinical stability. We have, therefore, taken the approach of using only those PEF data not measured within a period of 2 weeks before and 5 weeks after an exacerbation. Although one can debate indepth what arguments could enter the definition of clinically stable, such as lung function relative to predicted, airways hyperresponsiveness, sputum eosinophils/cytokines and exhaled nitric oxide, this approach, in retrospect of no exacerbations in the above-mentioned period, seems clinically feasible and relevant.

This report has focused entirely on overtreatment, whereas the guidelines have been perceived (but not tested) in an era of concern about undertreatment. This study shows the importance of the choice of the cut-off values. However, the choice of the correct cut-off values for self-management plans cannot be based on minimizing overtreatment alone. It should also be based on a minimum of undertreatment. In order to find these cut-off values, prospective studies have to be developed. From the perspective of overtreatment, it seems reasonable to test cut-off values $70-50 \%$ ( 2 days) for the yellow and red zone, respectively, expressed as percentage of PBTS. In these studies other definitions on the PB are also advisable to test, for instance the PB value achieved after maximal therapy or a regularly adjusted PBTS over time.

In summary, from the daily assessed peak expiratory flow values, morning peak expiratory flow measured before medication is the most useful parameter in self-management plans and should be related to the personal best value measured at the same time without prior use of a bronchodilator. Strict adherence to the 80 and $60 \%$ limits for the yellow and red zone, respectively, leads to a high prevalence of overtreatment in asthmatics who are treated with inhaled corticosteroids. There is no difference in the prevalence of overtreatment when using $60 \%$ personal best measured at 2 days as the limit for the red zone, or when using $50 \%$ measured on 1 day, taking into account the time of day and prior use of medication. The final choice of the correct cut-off value should be based on minimizing both overtreatment and undertreatment: not only should one try to prevent severe exacerbations (and deaths) but, for fear of exacerbations, one should not increase corticosteroids to ever higher dosages based on peak expiratory flow zone boundaries not thoroughly tested in everyday practice.

\section{References}

1. British Thoracic Society. Research Unit of the Royal College of Physicians of London, King's Fund Centre, National Asthma Campaign. Guidelines for management of asthma in adults: I. chronic persistent asthma. $\mathrm{Br} \mathrm{Med} J$ 1990; 301: 651-653.

2. British Thoracic Society. Research Unit of the Royal College of Physicians of London, King's Fund Centre, Natio- nal Asthma Campaign. Guidelines for management of asthma in adults: II. acute severe asthma. Br Med J 1990; 301: 797-800.

3. National Heart, Lung and Blood Institute, National Asthma Education Program, Expert Panel Report. Guidelines for the diagnosis and management of asthma. J Allergy Clin Immunol 1991; 88: (Suppl.), 425-534.

4. Brewis RAL. Patient education, self-management plans and peak flow measurement. Respir Med 1991; 85: 457-462.

5. International Asthma Management Project. International consensus report on the diagnosis and treatment of asthma. Bethesda, MD, NHLBI, NIH, 1992; publication no. 92-3091: pp. 1-72.

6. British Thoracic Society. Guidelines on the management of asthma. Thorax 1993; 48: Suppl., S1-S24.

7. Kotses H, Bernstein IL, Bernstein DI, et al. A self-manage-ment program for adult asthma. Part 1; Development and evaluation. J Allergy Clin Immunol 1995; 95: 529540.

8. Grampian Asthma Study of Integrated Care (GRASSIC). Effectiveness of routine self monitering of peak flow in patients with asthma. Br Med J 1994; 308: 564-567.

9. Taitel MS, Kotses H, Bernstein L, Bernstein DI, Creer TL. A self-management program for adult asthma. Part 2; Costbenefit analysis. J Allergy Clin Immunol 1995; 95: 672-676.

10. Lahdensuo A, Haahtela T, Herrala J, et al. Randomized comparison of guided self-management and traditional treatment of asthma over one year. Br Med J 1996; 312: $748-752$.

11. Ignacio-Garcia JM, Gonzalez-Santos P. Asthma self-management education program by home monitoring of peak expiratory flow. Am J Respir Crit Care Med 1995; 151: 353-359.

12. Meijer RJ, Kerstjens HAM, Postma DS. Guidelines and self-management plans in asthma. Eur Respir J 1997; 10: 1163-1172.

13. Kerstjens HAM, Brand PLP, Hughes MD, et al. A comparison of bronchodilator therapy with or without inhaled corticosteroid therapy for obstructive airways disease. $N$ Engl J Med 1992; 327: 1413-1419.

14. Brand PLP, Quanjer PH, Postma DS, et al. Interpretation of bronchodilator response in patients with obstructive airways disease. The Dutch Chronic Non-Specific Lung Disease (CNSLD) Study Group. Thorax 1992; 47: 429-436.

15. Quanjer PH. Standardized lung function testing. Bull Eur Physiopathol Respir 1983; 19: Suppl. 5, 1-95.

16. Brand PLP, Kerstjens HAM, Postma DS, et al. Long-term multicentre trial in chronic nonspecific lung disease: methodology and baseline assessment in adult patients. Eur Respir J 1992; 5: 21-31.

17. Cockcroft DW, Killian DN, Mellon JJA, Hargreave FE. Bronchial reactivity to inhaled histamine: a method and clinical survey. Clin Allergy 1977; 7: 235-243.

18. Brand PLP, Kersjens HAM, Janson HM, Kauffman HF, de Monchly JGR, Dutch CNCLD Study Group. Interpretation of skin tests to house dust mite and relationship to other allergy parameters in patients with asthma and chronic obstructive pulmonary disease. J Allergy Clin Immunol 1993; 91: 560-570.

19. Quanjer PH, Tammeling GL, Cotes JE, Pederson OF, Peslin R, Yernault YC. Lung volumes and forced ventilatory flows. Eur Respir J 1993; 6: Suppl. 16, 5-40.

20. Gibson PG, Wlodarczyk J, Henzley MJ, Murree-Allen K, Olson LG, Saltos N. Using quality control analysis of peak expiratory flow recordings to guide therapy for asthma. Ann Intern Med 1995; 123: 488-492. 